\title{
Evolution of Chi motifs in Proteobacteria
}

2

3

Angélique Buton \& Louis-Marie Bobay*

4

5 Department of Biology, University of North Carolina Greensboro, 321 McIver Street, PO Box

6 26170, Greensboro, NC 27402, USA

7

$8 *$ To whom correspondence should be addressed

9

10

11 Contact information

12 Louis-Marie Bobay

13321 McIver Street

14 Greensboro, NC 27402, USA

15 Email address: 1jbobay@uncg.edu

16 Phone: 336-256-2590

17

18 Keywords: homologous recombination, bacteria, genome evolution, DNA motifs.

19

20 


\section{Abstract}

Homologous recombination is a key pathway found in nearly all bacterial taxa. The recombination complex allows bacteria to repair DNA double strand breaks but also promotes adaption through the exchange of DNA between cells. In Proteobacteria, this process is mediated by the RecBCD complex, which relies on the recognition of a DNA motif named Chi to initiate recombination. The Chi motif has been characterized in Escherichia coli and analogous sequences have been found in several other species from diverse families, suggesting that this mode of action is widespread across bacteria. However, the sequences of Chi-like motifs are known for only five bacterial species: E. coli, Haemophilus influenzae, Bacillus subtilis, Lactococcus lactis and Staphylococcus aureus. In this study we detected putative Chi motifs in a large dataset of Proteobacteria and we identified four additional motifs sharing high sequence similarity and similar properties to the Chi motif of E. coli in 85 species of Proteobacteria. Most Chi motifs were detected in Enterobacteriaceae and this motif appears well conserved in this family. However, we did not detect Chi motifs for the majority of

35 Proteobacteria, suggesting that different motifs are used in these species. Altogether these results substantially expand our knowledge on the evolution of Chi motifs and on the recombination process in bacteria. 


\section{Introduction}

Bacteria frequently suffer DNA double-strand breaks; and these damages need to be efficiently repaired to avoid cell death. One of the primary repair mechanisms is mediated by homologous recombination which allows the exchange of homologous sequences and thus the repair of damaged DNA ${ }^{1}$. Homologous recombination is also a key mechanism contributing to the rapid evolution of bacteria and their viruses ${ }^{2}$. In Proteobacteria, the main enzymatic complex involved in this process is the RecBCD helicase-nuclease complex ${ }^{3,4}$. Following a doublestrand break, RecBCD binds to the broken end of the DNA and unwinds it ${ }^{5,6}$ upon encountering a Chi (Crossover Hotspot Instigator) site. The recognition of the Chi motif triggers an endonucleolytic nick near the 3 ' end of the Chi motif by $\operatorname{RecBCD}^{7}$ and initiates the loading of RecA proteins on the DNA strand ${ }^{8}$. The DNA-RecA complex can then initiate the search and exchange of genetic material with a homologous sequence ${ }^{7}$. The recognition of Chi motifs, mediated by the RecC subunit ${ }^{9}$, thus plays a key function in the initiation of the homologous recombination.

Chi sites have been well defined in Escherichia coli, where the sequence of this 8ntlong motif is 5'-GCTGGTGG-3' 10,11. This motif is over-represented on the genome with one motif every five kilobases on average ${ }^{12}$. Chi sites are also more frequent in the conserved regions of the bacterial genome (i.e. the core genome) than in the genes recently acquired ${ }^{13,14}$ and in genomic repeats ${ }^{15}$, suggesting the importance of the repair of the regions that encode the most essential cellular functions. This motif is also polarized on the chromosome, which is an important feature for the recognition of Chi sites by RecBCD ${ }^{16}$ : in E. coli Chi motifs are found almost exclusively on the leading strand of replication. Chi sites have an important role in the RecBCD pathway as its recognition by this enzymatic complex is a key step in initiating this mechanism. However, most of the knowledge on Chi sites has been derived from E. coli and little is known about this mechanism in other species. 

identified in four other bacteria from diverse taxonomic groups: Haemophilus influenzae (5'GNTGGTGG-3'/5'G(G/C)TGGAGG-3'), Bacillus subtilis (5'-AGCGG-3'), Lactococcus lactis (5'-GCGCGT-3') ${ }^{17}$ and Staphylococcus aureus (5'-GAAGCGG-3') ${ }^{13}$. Note that it remains unclear whether the 5'-AGCGG-3' motif stimulates homologous recombination in $B$. subtilis and that it can therefore be considered a true Chi motif. For this reason, we will refer to "Chi motifs" to designate the motifs found in E. coli and related bacteria with a similar sequence. In contrast, we will use "Chi-like motifs" to designate all the motifs that are thought to be associated with homologous recombination in prokaryotes. For most of the species with Chi-like sequences identified, Chi-like sequences are statistically over-represented in the core genome ${ }^{17}$. The presence of Chi-like motifs across highly divergent bacteria suggests that this mechanism of action is widespread in bacteria and possibly universal. Moreover, H. influenzae and E. coli are both Gammaproteobacteria and present related Chi sequences. Although Chilike sequences have likely evolved toward different sequences in most species, this last case suggests that they are evolving relatively slowly and indicates that it might be possible to identify them with computational approaches. across hundreds of species of Proteobacteria. By using sequence similarity, oligonucleotide frequency statistics and the known properties of Chi motifs (i.e. polarization on the leading strand and over-representation on the core genome) we detected the presence of candidate Chi motifs across 87 species of Proteobacteria. The identified motifs appear restricted to five sequences: (GCTGGTGG, GCTGGCGG, GCTGCTGG, GGTGGTGG and GCTGGAGG). We further used phylogenomic approaches to reconstruct the evolution of these sequences in

90 Proteobacteria. Our results underline that these sequences are well conserved in 91 Enterobactericeae, suggesting a common mode of action of homologous recombination in these 

most species, since most species present a single or a few sequenced genome(s). To circumvent

species. However, we did not find related sequences across most Proteobacteria, indicating that Chi motifs are either absent in these lineages or possess highly divergent sequences.

\section{Materials and Methods}

\section{Data collection}

Genomic sequences were downloaded from GenBank in May 2018. One genome per species was selected. If multiple genomes were available, we selected the one with the most complete assembly (the one with the smallest number of contigs), and if a species presented multiple assemblies with the same quality, we randomly selected one of the genomes. Following this procedure, we obtained 8,924 genomes from distinct species. From this list, we extracted the species belonging to the Proteobacteria and the Terrabacteria, which yielded a total of 2603 species, composed of 1,071 and 992 species of Proteobacteria and Terrabacteria, respectively. We then selected the genomes that with complete assemblies (i.e. assemblies at the scaffold or the contig level were discarded), and this yielded a total or 495 Proteobacteria and 363 Terrabacteria. Dataset information such as species name, GenBank accession number, taxonomy, genome size and nucleotide composition (GC-content) are detailed in Dataset S1.

\section{Definition of the core genome}

It has been previously observed that Chi sites are only statistically over-represented on the core genome ${ }^{17}$, likely because these regions need to be repaired efficiently. In order to improve the sensitivity of our approach, we restricted our analyses of Chi motifs to the core genome of each species. The core genome of a bacterial species is typically defined by all the genes present in every or almost every strain of a species. However, it is not possible to build a core genome for this issue, we built a pseudo core genome for each species by using the set of orthologous genes 
117 shared among closely related species. Indeed, the core genes of a given species typically encode

118 for the most essential cellular functions (typically housekeeping genes) and those are expected

119 to be shared by closely related species. For each species, we used HMMer v3 ${ }^{18}$ and the set of

12043 universal protein profiles identified in a previous study ${ }^{19}$. Each of the universal proteins

121 identified were extracted and aligned with MAFFT v7 ${ }^{20}$. For each alignment, poorly aligned

122 regions were removed using Gblocks v0.91b ${ }^{21}$. The 43 universal protein alignments were then

123 concatenated into a single alignment. All pairwise distances were computed on the concatenate

124 and the distances were used to identify all the pairs of most closely related species. For each

125 pair of most related species, the set of shared orthologs was defined as best reciprocal hits using

126 USEARCH v11 (global alignment) with a minimum of $70 \%$ sequence identity and $80 \%$ length

127 conservation ${ }^{22}$. For each species, the pseudo core genome was defined as the set of genes shared

128 by the two most closely related species.

\section{Detection of Chi sites}

131 Candidate Chi motifs were inferred by using multiple criteria. First, we searched for the

132 presence of candidates Chi sites with an identical or highly similar sequence of the motif found

133 in E. coli (5'-GCTGGTGG-3'). All the motifs with one nucleotide difference compared to the

134 motif od E. coli have been analyzed, namely a total of 25 motifs: GCTGGTGG, ACTGGTGG,

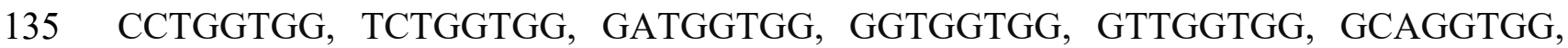

136 GCGGGtGG，GCCGGtGG，GCtAGtGG，GCTTGTGG，GCTCGTGG，GCTGATGG,

137 GCtGTtGG, GCTGCTGG, GCtGGAGG，GCTGGCGG，GCTGGGGG，GCTGGTAG，

138 GCTGGTCG, GCTGGTtG, GCTGGTGA, GCTGGTGC, GCTGGTGT. The pseudo core

139 genomes of the 2,063 species were analyzed by the software R'MES v3.1.0 ${ }^{23}$. The statistics of

140 all the possible octamers were performed using a Gaussian law ${ }^{24-26}$ in R'MES v3.1.0, allowing

141 the calculation of the expected frequency of the motifs relative to the oligonucleotide 
composition of the genomes. The over-representation of each motif was then evaluated by a

143 statistical comparison between the observed and expected numbers using different Markov

144 models to take into account the different levels of oligonucleotide composition of the sequences

145 (different composition bias: mono-nucleotides, di-nucleotides, tri-nucleotides, quadra-

146 nucleotides and penta-nucleotides and hexa-nucleotides). For each model, a motif was

147 considered as significantly overrepresented based on the statistics observed for the Chi site

148 frequencies of E. coli (thresholds used are presented Table S1). The detected motifs were

149 considered as candidate Chi motifs when found significantly over-represented in the core

150 genome with at least three different Markov models and when found polarized on the genome.

151 The polarization statistics were computed with the Wilcoxon signed-rank test implemented in

152 R version 3.5.1. Since we couldn't confidently identify the origin and terminus of replication

153 for all species, we reasoned that, in the absence of polarization on the genome, Chi sites should

154 be randomly distributed on both DNA strands. If Chi sites are polarized on the chromosome,

155 we should observe a biased distribution where consecutive Chi sites should be found on the

156 same strand more frequently than expected by chance. We compared the distribution of the

157 positions of Chi sites on the positive strand and on the negative strand. We considered that the

158 candidate Chi motifs were polarized on the genome when the distribution was significantly

159 biased ( $p$-value $<0.001$, Wilcoxon test) from the null expectation.

160

\section{Phylogenetic analysis}

162 The concatenated alignment of 43 protein sequences described above was used to build the

163 phylogenetic tree of the Proteobacteria ${ }^{19}$. We built the phylogenetic tree with a maximum

164 likelihood approach using RAxML v8 ${ }^{27}$ with the LG model, which was selected as best-scoring

165 model by RAxML. The tree was rooted by including the sequences of three Firmicutes: Bacillus 
cereus, Lactobacillus casei and Staphylococcus saprophyticus. The resulting tree was edited with colors and legends with iTOL ${ }^{28}$. The Newick format of the tree is available in Dataset S2.

\section{Results}

Detection of five Chi motifs in Proteobacteria.

171 We used multiple criteria to detect putative Chi sites in our dataset of 495 Proteobacteria: i)

172 sequence similarity to the Chi motif of E. coli, ii) polarization with the leading strand of

173 replication and iii) statistical overrepresentation on the core genome relative to the

174 oligonucleotide composition of these genomes (see Methods for details). Because multiple

175 oligonucleotide biases can impact the inference of Chi motifs, we computed the statistical

176 overrepresentation of these motifs in the core genomes with R'MES using different Markov

177 models: from model 0 , which accounts for the mononucleotide composition of the core genome,

178 to model 6, which accounts for the pentanucleotide composition of the core genome. A Chi

179 motif was inferred as a candidate when statistically overrepresented in the core genome with

180 three Markov models (see Table S2-3 for the detailed results across all Markov models). Using

181 these different criteria, we identified 85 species of Proteobacteria with candidate Chi sites (see

182 Dataset S3 for details). Across these species, five main motifs were identified as potential Chi

183 motifs in Proteobacteria: GCTGGTGG, GCTGGCGG, GCTGCTGG, GGTGGTGG and

184 GCTGGAGG (Figure 1). The most frequent motif, GCTGGTGG, is the Chi motif shown to be 185 functional in E. coli.

187 Estimation of false positives.

188 To assess the quality of our detection approach, we used a large sample of Terrabacteria $(\mathrm{n}=363)$

189 to estimate our rate of false positive detection (see Table S1-3 for the detailed results across all

190 Markov models). Terrabacteria are highly divergent from Proteobacteria but they also exhibit a 
191 large diversity of genome size and nucleotide composition. We reasoned that these divergent

192 bacteria are not expected to present similar motifs to Proteobacteria. Indeed, the Chi motifs

193 previously identified in B. subtilis, L. lactis and S. aureus are not related to the motifs inferred

194 in Proteobacteria. Using the same detection procedure for the 25 putative motifs, we estimated

195 our rate of false positives to be 3.9\%. Interestingly, the putative Chi motifs are GC-rich and the

196 false positives were mostly found in species of Terrabacteria with high GC-content, indicating

197 that a weak detection bias due to sequence composition remains even though our framework

198 should compensate for mononucleotide and oligonucleotide compositions.

\section{GC-content and Chi sites}

201 The reference Chi motif of E. coli is a GC-rich sequence and all the related Chi motifs that we searched in these genomes present high GC-content (76.7\% on average). We observed that the species with inferred candidate Chi motifs present higher genomic GC-content (60.8\%) relative

to the species without Chi motifs (respectively, $60.8 \%$ and $51.5 \%$; $P<10^{-5}$; Wilcoxon test;

Figure S1A). This difference was also observed when limiting the comparison to

206 Gammaproteobacteria $\left(P<10^{-5}\right.$; Wilcoxon test; Figure S1B). However, the genomic GC-content 207 of Proteobacteria is much lower than the GC-content of Chi motifs (respectively, 60.8\% and $20876.6 \% ; P<10^{-5}$; Wilcoxon test; Figure S1A). These results suggest that species harboring these

209 Chi motifs tend to be relatively GC-rich, but their GC-content remains much lower than the 210 GC-content of the motif itself.

\section{Evolution of Chi sites in Proteobacteria.}

213 We further analyzed the evolution of Chi motifs in Proteobacteria. To do so, we reconstructed

214 the phylogenetic tree of Proteobacteria using a set 43 universal proteins previously identified

215 19. The distribution of species with inferred Chi motifs in the Proteobacteria tree confirms that 
216 the vast majority of these species are close relatives of E. coli: mostly Enterobacteriaceae

217 (Gammaproteobacteria) (Figure 2). Several species of Gammaproteobacteria-other than the

218 Enterobacteriaceae - were inferred to possess candidate Chi motifs (Aeromonas, Zobellella,

219 Pseudomonas et Kushneria) but their genomes contain a GC-content of about 60\%, which

220 suggests that these could represent false positives. In the other groups of Proteobacteria, we

221 detected Chi motifs among Betaproteobacteria (Paraburkholderia genus), as well as in

222 Epsilon/Deltaproteobacteria (Myxococcus genus) and Alphaproteobacteria (Azorhizobium

223 caulinodans and Komagataeibacter europaeus). Those species all present a relatively high GC-

224 content $(\mathrm{GC} \%>60 \%)$, also suggesting that the Chi motifs inferred in these species likely

225 represent false positive. For comparison, the GC-content of E. coli is $52.3 \%$.

226 In contrast, virtually all Enterobacteriaceae presented Chi motifs. One notable

227 exception is the genus Yersinia for which we did not detect the presence of Chi sites for any of

228 the available species (Figure 3). The phylogenetic distribution of species with inferred Chi sites

229 suggests that this motif was present in the ancestor of all Enterobacteriaceae and was

230 subsequently lost in Yersinia and several Serratia species. Alternatively, it is possible that Chi

231 sites were acquired after the divergence of the Yersinia/Serratia lineage and subsequently

232 transferred to Serratia proteamaculans. Interestingly, many species of Enterobacteriaceae

233 possess multiple Chi motifs. Indeed, three motifs are frequently identified in the same species:

234 GCTGGTGG, GCTGGCGG and GCTGCTGG. This suggests that several species might

235 recognize the degenerated Chi motif GCTG $[\mathrm{G} / \mathrm{C}][\mathrm{T} / \mathrm{C}] \mathrm{GG}$. Finally, some of the more basally

236 branching species of Enterobacteriaceae such as members of the genera Dickeya and

237 Edwardsiella were predominantly found to present the motif GGTGGTGG. These results

238 suggest, that Chi motifs are relatively well conserved across Enterobacteriaceae but that these

239 sequences have experienced some minor sequence changes over time. 


\section{Extending the search of Chi motifs}

242 Our initial analysis was based on stringent parameters to identify candidate Chi sites with high

243 confidence using the same properties observed for E. coli's Chi sites. It is very likely, however,

244 that some Chi sites do not possess similar properties in other species. We conducted the same

245 detection strategy without imposing any constraints on sequence polarity. This extended our

246 list to a total of 234 species of Proteobacteria with candidate Chi motifs (Dataset S4) and these

247 motifs were found in 64 Terrabacteria (21\% of the species). Although this likely increases our

248 rate of false positives, it is possible that many of these candidate motifs are true Chi motifs that

249 need not be polarized to be functional.

We then extended the search of putative Chi motifs to more divergent sequences.

251 Starting from the five candidate motifs that we detected, we generated 830 additional non-

252 redundant motifs, each containing two different nucleotides relative to one of these five motifs.

253 The same procedure was then used to search for statistically overrepresented motifs based on

254 oligonucleotide compositions on the core genome and sequence polarity. We found very few

255 polarized motifs statically overrepresented relative to oligonucleotide compositions of the

256 genomes (Table S4). Using our previous criteria, we did not identify any of these motifs to be

257 polarized and statistically overrepresented using at least three Markov models (Table S5).

258 Finally, we searched for these 830 motifs without the polarity criterion and this yielded a list of

259 only 11 species of Proteobacteria with candidate Chi motifs for 16 species of Terrabacteria

260 (Dataset S5), indicating that these motifs are likely false positives. This result further suggests

261 that reducing the stringency of our parameters does not substantially increase the list of species

262 with candidate Chi motifs. 


\section{Discussion}

267 This study suggests that a relatively small proportion of Proteobacteria use Chi motifs. We 268 found that a minority of Gammaproteobacteria (12.5\% of the total Proteobacteria) is likely 269 using this Chi motif. However, this study was limited to a list of motifs closely related to the one found in $E$. coli, i.e. a list of motifs with a one nucleotide difference (25 motifs). Among

271 the 25 sequences, only five were found frequently in the Proteobacteria: 5'-GCTGGTGG-3', 5'-GCTGGCGG-3', 5'-GCTGCTGG-3', 5'-GGTGGTGG-3' and 5'-GCTGGAGG-3'. it is possible that some of these species of Proteobacteria do not have Chi sites. Indeed, some of 274 these species completely lack (e.g. Buchnera aphidicola) or show a strong reduction in 275 homologous recombination (e.g. Yersinia pestis) ${ }^{29}$. In addition, some species of Proteobacteria, such as Alphaproteobacteria, rely on the AddBC recombination system instead of RecBCD ${ }^{4}$.

277 However, given the focus of our procedure on Chi sites related to E. coli's motif, it is very likely that more divergent—or unrelated — Chi-like sequences exist in Proteobacteria. Searching for additional motifs could enable the identification of Chi sites in these species. This endeavor 280 remains difficult due the sensitivity of ab initio detections and that no Chi-like motifs unrelated to E. coli's motif have been identified in Proteobacteria so far. In addition, it may not be possible at all to detect such motifs using computational approaches if these sequences are simply not statistically over-represented in the genome (see below).

Given the large dataset used for this study, it is likely that some Chi motifs identified correspond to detection errors (i.e. false positives). By using a large set of Terrabacteria species, which are thought to use different motifs ${ }^{17}$, we identified our rate of false positives to reach

287 about $4 \%$ of detected motifs. The detection of false positives seems to be closely linked to the 288 richness in GC-content since most false positives were found in GC-rich genomes (e.g. 289 Actinobacteria). Although the nucleotide composition biases were taken into account by the 290 statistics generated by R'MES, it seems that these filters did not completely eliminate all the 
false positives due to nucleotide composition. This suggests that the candidate Chi motifs inferred in GC-rich species should be taken with care.

293 The evolution of Chi sites and other DNA motifs is still poorly understood; however, we can

294 conclude that species related to $E$. coli likely use very similar motifs, indicating that these motifs

295 have been conserved for millions of years. Based on our phylogenetic analysis we can infer that

296 this motif appeared at least since the divergence between the Enterobacteriaceae and the rest 297 of the Gammaproteobacteria and that the sequence of the motif evolved in some of these taxa.

298 In agreement with our results, it was previously observed that several species of

299 Enterobacteriaceae were capable of Chi-dependent cleavage of DNA constructs containing $E$.

300 coli's Chi motif ${ }^{30,31}$. Our results extend these findings by showing that most

301 Enterobacteriaceae contain polarized and statistically overrepresented Chi motifs in their core

302 genomes and that some of these species likely use slightly different motifs than E. coli's motif

303 (or a degenerated version of this motif). However, we found that the majority — but not all—

304 species of Enterobacteriaceae use Chi motifs similar to E. coli's motif. Indeed, we did not 305 detect the presence of polarized Chi motifs in Vibrionaceae, although several of these species 306 were shown capable of Chi-dependent cleavage of DNA constructs containing E. coli's Chi 307 motif ${ }^{30}$. However, we identified a non-polarized candidate Chi motif (GCTGGTGG) in 23 308 species of Vibrio (Dataset S4), indicating that polarity might not be required for the proper 309 function of Chi motifs in these species. We did not detect candidate Chi motifs in H. influenzae 310 (Pasteurellaceae), although Chi motifs were identified for this species in a previous study ${ }^{17}$. 311 In fact, the original study that identified Chi sites in $H$. influenzae ${ }^{17}$ showed that these motifs 312 are degenerated, less represented statistically and less polarized than the ones found in E. coli, 313 even though their function has been experimentally validated ${ }^{32}$. We used stringent criteria to 314 avoid the detection of false positives, but it is possible that some species present Chi motifs too 315 degenerated to be confidently identified using in silico approaches. Furthermore, it is likely that 
316 other species of Proteobacteria contain Chi sites with other sequences than those we searched

317 for in this study. It is possible that the stringency of our approach prevented us from identifying

318 additional motifs but relaxing our search criteria directly leads to a much higher rate of false

319 positives. Our results do not constitute a proof that most Proteobacteria are devoid of Chi or

320 Chi-like motifs, but our analyses rather suggest that their putative motifs must substantially

321 differ from E. coli's motif. Considering that some experimentally characterized motifs, such as

322 the ones of $H$. influenzae, virtually escape to all bioinformatic detection procedure, it is possible

323 that only an experimental approach would allow to confidently detect new Chi-like motifs in

324 Proteobacteria. Together, these results contribute to better characterizing the signals and

325 pathways involved in homologous recombination in bacteria, as well as the evolution of

326 genomic motifs.

Overall, our results indicate that Chi motifs similar to E. coli's motif evolved at least since the ancestor of Enterobacteriaceae and were lost in several lineages. Some of these species likely use motifs whose sequence is slightly different from E. coli's motif. Using in silico approaches we did not confidently detect such motifs in other species of Proteobacteria, suggesting that these lineages use unrelated sequences or that these motifs do not present all the characteristics of E. coli's motif (i.e. polarization on the leading strand). Experimental analyses will be needed to confirm that these motifs can efficiently trigger RecBCD-mediated

334 homologous recombination. Overall this study revealed new candidate Chi motifs and extends 335 our understanding of the mechanisms controlling homologous recombination in Proteobacteria. 


\section{Acknowledgments}

341 We thank Shealynn Burgess for assistance with the analyses, Kasie Raymann for criticisms on

342 the manuscript and Caroline Stott for assistance with the figures. This work was supported by

343 the National Science Foundation under Grant No. DEB-1831730 by the National Institute Of

344 General Medical Sciences of the National Institutes of Health under Award Number 345 R01GM132137.

347 Authors' contributions

$348 \mathrm{LMB}$ and $\mathrm{AB}$ designed the study. $\mathrm{LMB}$ and $\mathrm{AB}$ analyzed the data. $\mathrm{LMB}$ and $\mathrm{AB}$ wrote the

349 manuscript. All authors read and approved the final manuscript. 


\section{References}

3521 Resnick, M. A. The repair of double-strand breaks in DNA; a model involving recombination. J Theor Biol 59, 97-106 (1976).

3542 Bobay, L. M., Rocha, E. P. \& Touchon, M. The adaptation of temperate bacteriophages to their host genomes. Mol Biol Evol 30, 737-751 (2013).

3563 McKittrick, N. H. \& Smith, G. R. Activation of Chi recombinational hotspots by RecBCD-like enzymes from enteric bacteria. J Mol Biol 210, 485-495 (1989).

Rocha, E. P. C., Cornet, E. \& Michel, B. Comparative and evolutionary analysis of the bacterial homologous recombination systems. PLoS Genet 1, e15 (2005).

3605 Taylor, A. \& Smith, G. R. Unwinding and rewinding of DNA by the RecBC enzyme. Cell 22, 447-457 (1980). RecBCD. Elife 5, doi:10.7554/eLife.18227 (2016).

3647 Smith, G. R. How RecBCD enzyme and Chi promote DNA break repair and recombination: a molecular biologist's view. Microbiol Mol Biol Rev 76, 217-228 (2012).

Anderson, D. G. \& Kowalczykowski, S. C. Reconstitution of an SOS response pathway: derepression of transcription in response to DNA breaks. Cell 95, 975-979 (1998). stranded DNA regulatory sequence, chi, by RecBCD enzyme. Proc Natl Acad Sci U S

37210 Smith, G. R., Kunes, S. M., Schultz, D. W., Taylor, A. \& Triman, K. L. Structure of chi hotspots of generalized recombination. Cell 24, 429-436 (1981). 
37411 Smith, G. R., Comb, M., Schultz, D. W., Daniels, D. L. \& Blattner, F. R. Nucleotide sequence of the chi recombinational hot spot chi $+\mathrm{D}$ in bacteriophage lambda. $J$ Virol 37, 336-342 (1981).

37712 Touzain, F., Petit, M. A., Schbath, S. \& El Karoui, M. DNA motifs that sculpt the bacterial chromosome. Nat Rev Microbiol 9, 15-26 (2011).

37913 Halpern, D. et al. Identification of DNA motifs implicated in maintenance of bacterial core genomes by predictive modeling. PLoS Genet 3, 1614-1621 (2007).

38114 Cockram, C. A., Filatenkova, M., Danos, V., El Karoui, M. \& Leach, D. R. Quantitative genomic analysis of RecA protein binding during DNA double-strand break repair reveals RecBCD action in vivo. Proc Natl Acad Sci U S A 112, E4735-4742 (2015).

38415 Li, C. et al. The positioning of Chi sites allows the RecBCD pathway to suppress some genomic rearrangements. Nucleic Acids Res 47, 1836-1846 (2019). at Chi sites during DNA unwinding: location and orientation-dependence of the cutting. Cell 41, 153-163 (1985).

38917 El Karoui, M., Biaudet, V., Schbath, S. \& Gruss, A. Characteristics of Chi distribution on different bacterial genomes. Res Microbiol 150, 579-587 (1999).

$391 \quad 18$ Eddy, S. R. Accelerated Profile HMM Searches. PLoS Comput Biol 7, e1002195 (2011).

39219 Raymann, K., Brochier-Armanet, C. \& Gribaldo, S. The two-domain tree of life is 393 linked to a new root for the Archaea. Proc Natl Acad Sci U S A 112, 6670-6675 (2015).

$39420 \quad$ Katoh, K. \& Standley, D. M. MAFFT multiple sequence alignment software version 7: 395 improvements in performance and usability. Mol Biol Evol 30, 772-780 (2013).

39621 Castresana, J. Selection of conserved blocks from multiple alignments for their use in phylogenetic analysis. Mol Biol Evol 17, 540-552 (2000). 
39822 Edgar, R. C. Search and clustering orders of magnitude faster than BLAST. Bioinformatics 26, 2460-2461 (2010).

40023 Schbath, S. \& Hoebeke, M. R'MES: a tool to find motifs with a significantly unexpected frequency in biological sequences. World Scientific edn, Vol. 7 25-64 (World Scientific, 2011).

40324 Schbath, S., Prum, B. \& Turckheim, E. d. Exceptional motifs in different Markov chain models for a statistical analysis of DNA sequences. J Comput Biol 2, 417-437 (1995). sequences. J Comput Biol 4, 189-192 (1997). in deoxyribonucleic acid sequences. J. Royal Stat. Soc 57, 205-220 (1995).

27 Stamatakis, A. RAxML version 8: a tool for phylogenetic analysis and post-analysis of large phylogenies. Bioinformatics 30, 1312-1313 (2014).

41128 Letunic, I. \& Bork, P. Interactive Tree Of Life (iTOL): an online tool for phylogenetic tree display and annotation. Bioinformatics 23, 127-128 (2007).

41329 Cui, Y. \& Song, Y. Genome and evolution of Yersinia pestis. Adv Exp Med Biol 918 $414 \quad$ (2016).

$41530 \quad$ Schultz, D. W. \& Smith, G. R. Conservation of Chi cutting activity in terrestrial and marine enteric bacteria. J Mol Biol 189, 585-595 (1986).

41731 Smith, G. R., Roberts, C. M. \& Schultz, D. W. Activity of Chi recombinational hotspots 418 in Salmonella typhimurium. Genetics 112, 429-439 (1986).

41932 Sourice, S., Biaudet, V., Karoui, M. E., Ehrlich, S. D. \& Gruss, A. Identification of the Chi site. Mol Microbiol 27, 1021-1029 (1998). 


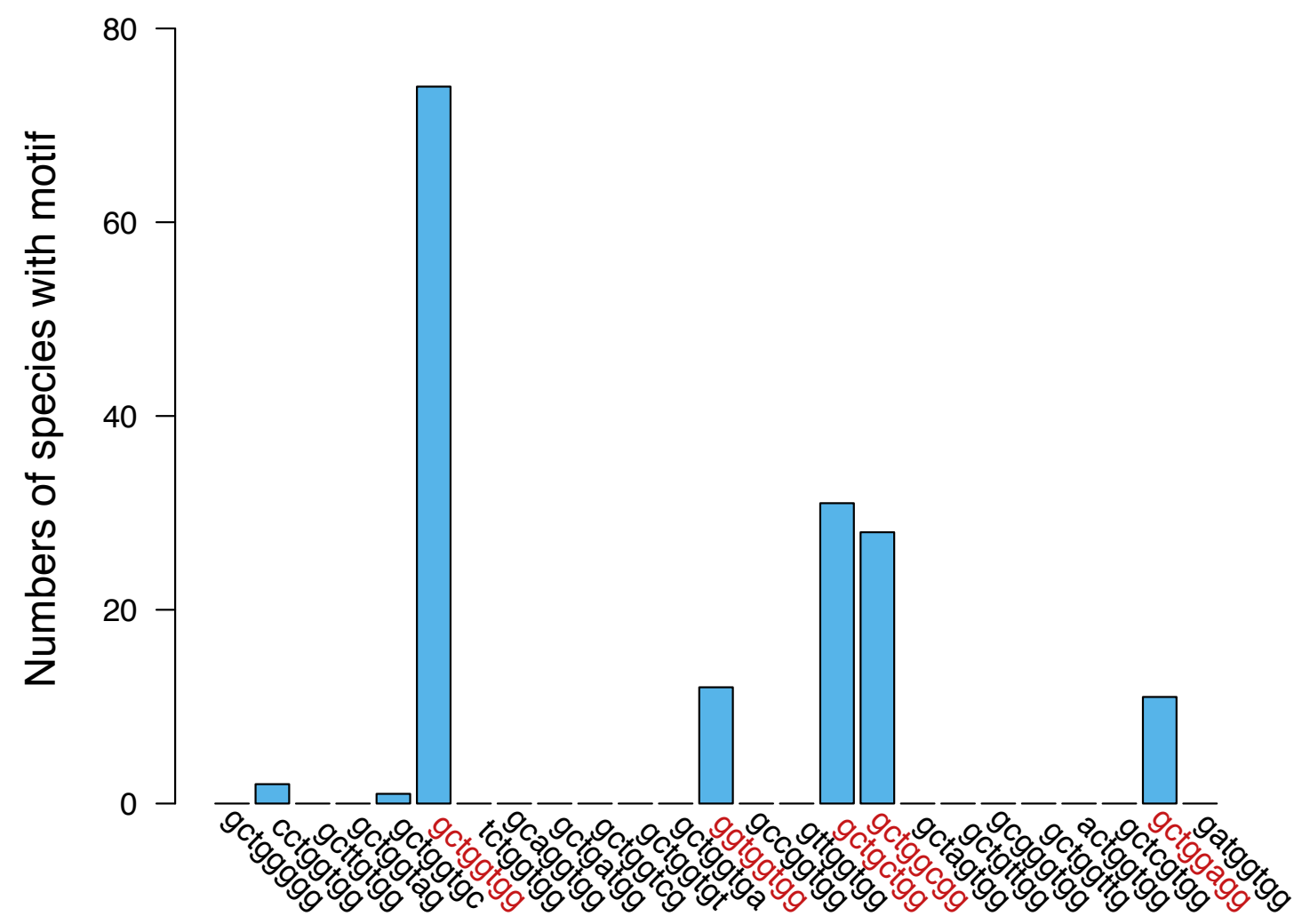

425 Figure 1: Frequencies and sequences of candidate Chi motifs identified in Proteobacteria.

426 Five main Chi motifs have been detected in a total of 87 species of Proteobacteria based on our 427 criteria. 


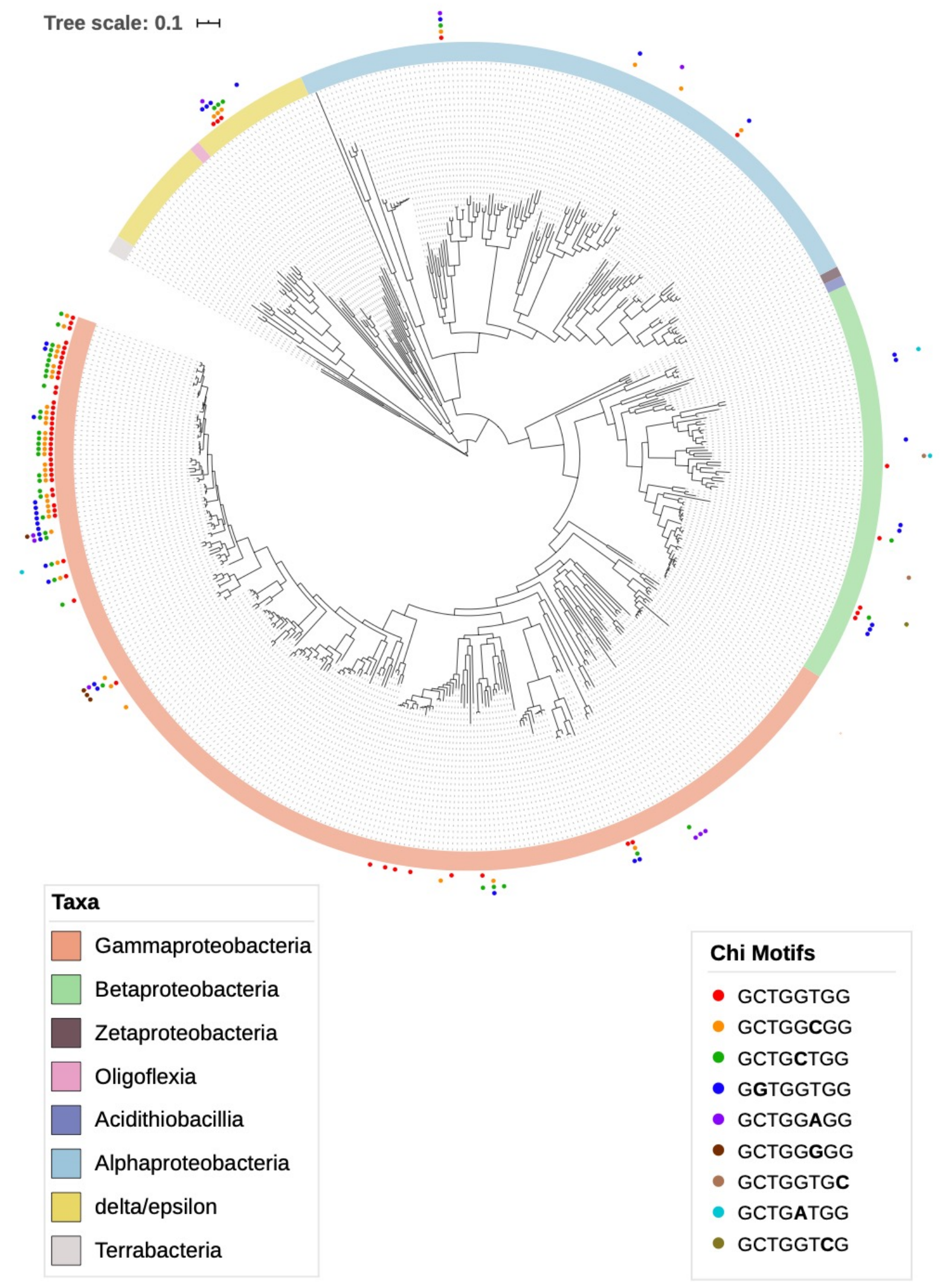

$430 \quad$ Figure 2: Phylogenetic tree of Proteobacteria and distribution of species with inferred Chi 
bioRxiv preprint doi: https://doi.org/10.1101/2020.08.13.249359; this version posted August 14, 2020. The copyright holder for this preprint (which was not certified by peer review) is the author/funder, who has granted bioRxiv a license to display the preprint in perpetuity. It is made available under aCC-BY-NC-ND 4.0 International license.

431 motifs. The tree was inferred using a set of 43 universal proteins and with RaxML (see

432 Methods) using the LG substitution model and rooted using three Firmicutes species.

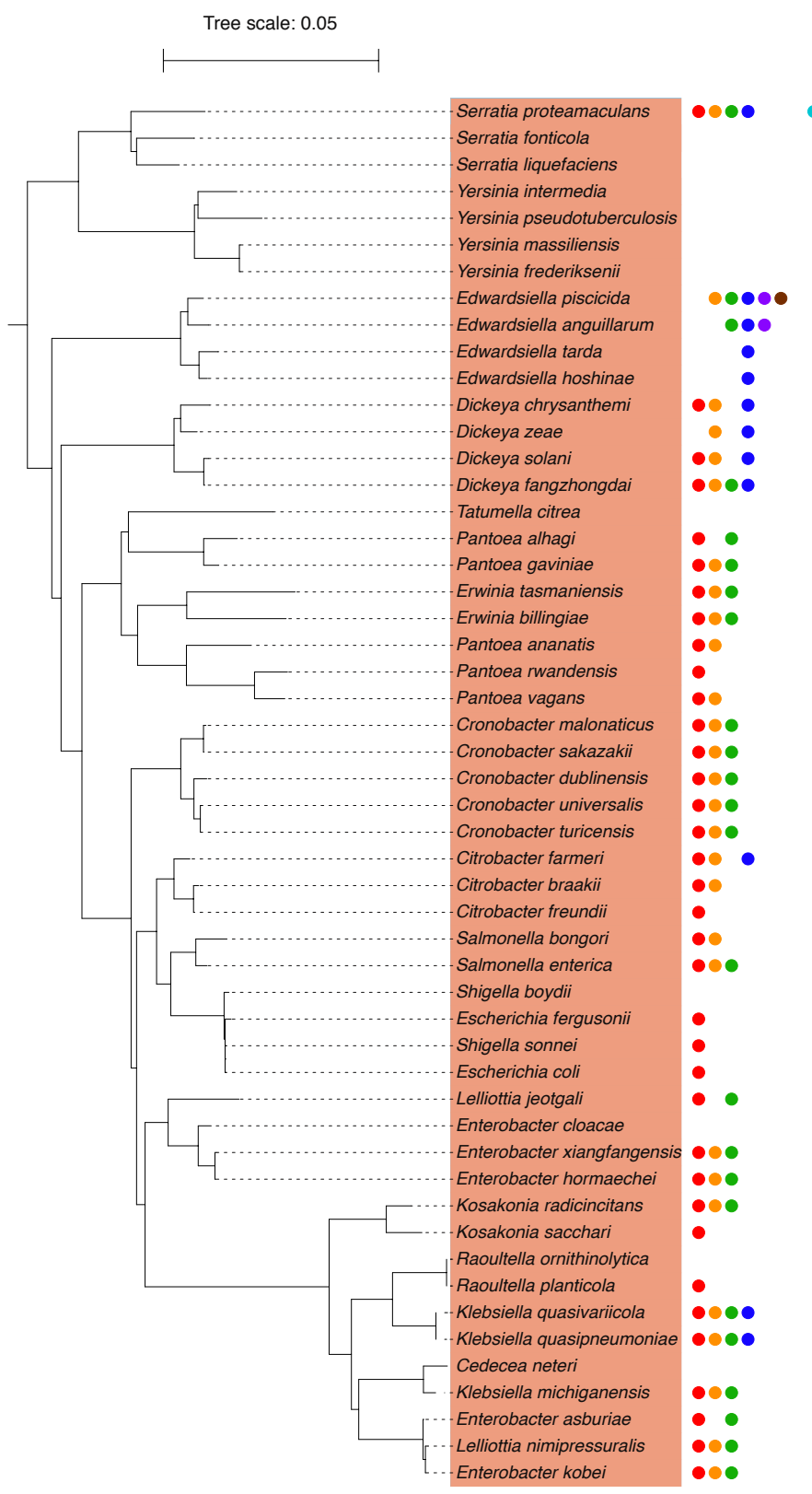

436 Figure 3: Distribution of candidate Chi motifs in Enterobacteriacaea. The subtree was 437 extracted from the tree of Proteobacteria presented in Figure 2 and rooted with non$438 \quad$ Enterobacteriacaea species. 
A

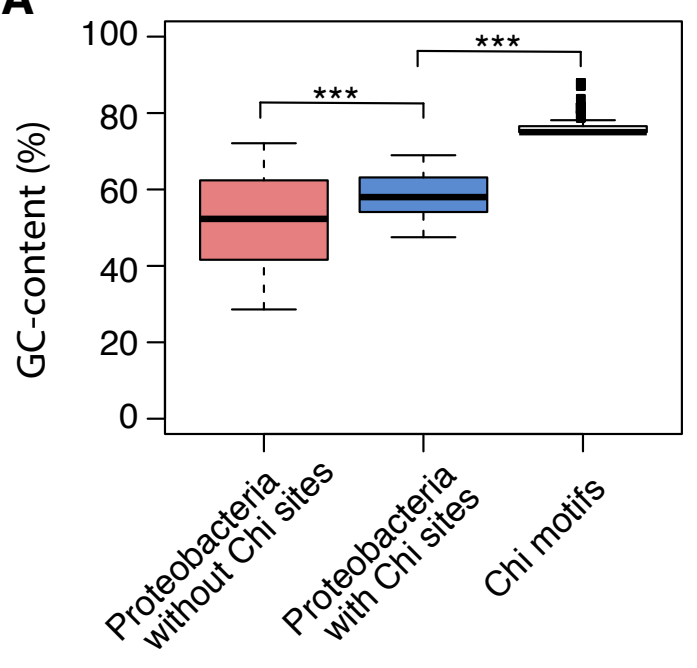

B

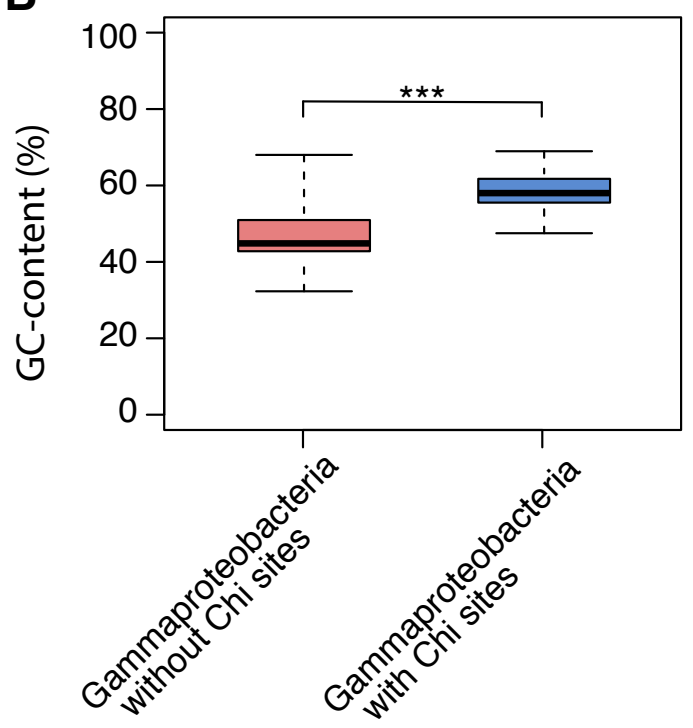

440 Figure S1: GC-content of Proteobacteria with or without inferred Chi sites. A comparison

441 of GC-content between species of Proteobacteria with inferred Chi sites and without inferred

442 Chi sites. The GC-content of the candidate Chi motifs is indicated in the third boxplot. B

443 comparison of GC-content between species of Gammaproteobacteria with inferred Chi sites 444 and without inferred Chi sites. (***) $P<10^{-5}$, Wilcoxon test. 
Supplementary Tables and Datasets

447

Table S1. Statistical overrepresentation of Chi motifs across Markov models.

Table S2. Number of species identified with Chi motifs across Markov models (25 motifs

451 searched).

452

Table S3. Number of species identified with Chi motifs 25 motifs searched).

Table S4. Number of species identified with Chi motifs across Markov models (830 motifs searched).

Table S5. Number of species identified with Chi motifs (830 motifs searched).

Dataset S1. List of analyzed genomes and genomic features.

Dataset S2. Phylogenetic tree of Proteobacteria in Newick format.

464 Dataset S3. List of species with candidate Chi motifs (25 polarized motifs searched).

466 Dataset S4. List of species with candidate Chi motifs (25 non-polarized motifs searched). 